\title{
1 Analysis of fracture healing process by HR-pQCT in patients with distal radius
}

\section{2 fracture}

3

\section{Abstract}

$5 \quad$ Introduction

6 High-resolution peripheral quantitative computed tomography (HR-pQCT) has enabled

7 us to observe the changes in bone microarchitecture over time in vivo. In this study, the

8 process of fracture healing was analyzed using HR-pQCT in patients with distal radius

9 fracture who underwent osteosynthesis.

\section{Methods}

11 A total of 10 fracture sites identified from four patients with a distal radius fracture who

12 underwent internal fixation with a volar locking plate (mean age 68.8 years, all women)

13 were investigated. HR-pQCT was performed within a week (baseline), 4, 12, and 24

14 weeks after fracture. Rectangular region of interest (ROI) was established in the fracture

15 site, inner callus, and external callus area, and the changes in bone mineral density

16 (BMD) in each region were analyzed.

\section{Results}

18 From baseline to 24 weeks post-fracture, the BMD changed from 105.5 (95\%CI 98.6-

$19113)$ to $428.0(331-554) \mathrm{mgHA} / \mathrm{ccm}$ at the fracture site, from $111.0(104-119)$ to 375.3

20 (290-486) $\mathrm{mgHA} / \mathrm{ccm}$ at the inner callus area, and from $98.5(91.6-106)$ to $171.6(132-$

21 222) $\mathrm{mgHA} / \mathrm{ccm}$ at the external callus area. The BMD increased at the fracture site and inner callus area, but increased only slightly at the external callus area. At 24 weeks 
23 post-fracture, the BMD at the fracture site and inner callus area was significantly higher

24 than the external callus area.

\section{Conclusion}

26 In the healing process of postoperative distal radius fractures, increased BMD at the

27 inner surface of the fracture site was confirmed in all fractures. Bone formation on the

28 endosteal side may be a necessary condition for bone union of distal radius fractures.

29

30

31

32 Running title: Fracture healing process analyzed by HR-pQCT

33

34 Keywords: fracture healing; high-resolution peripheral quantitative computed tomography (HR-pQCT); distal radius fracture 


\section{Introduction}

38 The fracture healing process is classified into four stages: an inflammatory stage, soft

39 calluses formation stage, hard calluses formation stage, and remodeling stage $[1,2]$.

40 Inflammatory stage occurs soon after a fracture. By releasing powerful cytokines

41 accompanying destruction of platelets, hematoma replaced with granulation tissue.

42 Then, a soft callus formation occurs with membranous ossification. Hard callus

43 formation begins after continuity of the fractured ends with soft calluses. Remodeling

44 begins when the fracture site is tilled with woven bone.

45 The analyses of fracture healing process have been performed basically with animal

46 experiments $[3,4]$. However, microlevel analyses of the fracture healing in the living

47 human body have been challenging due to limited resolution of imaging modality.

48 Methods to evaluate fracture healing by clinical imaging modality include plain X-

49 ray and clinical computed tomography (CT). An assessment with plain X-ray is two-

50 dimensional, and the status of fracture healing cannot be analyzed in detail. On the other

51 hand, clinical CT contributes assessment of fracture healing more in detail three-

52 dimensionally [5], however, microlevel observation of the fracture healing was

53 challenging.

54 High-resolution peripheral quantitative CT (HR-pQCT) is a clinical CT dedicated to

55 human extremities. It has the highest resolution in the clinical CT (voxel size of 60.7

$56 \mu \mathrm{m}$ ), enabling us to observe the changes in bone microarchitecture in the human body

57 over time with a low exposure dose (approximately $5 \mu \mathrm{Sv}$ of effective dose for $1 \mathrm{~cm}$ )

$58[6-8]$. 
59 Observations of the fracture healing process using HR-pQCT have been reported in

60 cases of conservative treatment for stable distal radius fractures [9-11]. However,

61 research on the healing process after surgical treatment for unstable distal radius

62 fractures was challenging because it was affected by metal artifacts.

63 Era et al. reported the method of assessing the distal radius after osteosynthesis by

64 HR-pQCT, showing acceptable accuracy (0.3-1.7 \% of difference in cadaveric bone

65 with and without metal plate) and precision (1.1\% of root-mean-square percent

66 coefficient of variation: $\mathrm{RMS} \% \mathrm{CV}$ ) [12]. In this method, the scan region is restricted at

67 the middle of metal plate where there are no screws in the bone. Also, the measurement

68 region is restricted at the dorsal one-third of the cortical bone, resulting less susceptible

69 assessment to the metal artifacts.

70 In this study, we observed the fracture site of patients who underwent osteosynthesis

71 for unstable distal radius fractures over time using HR-pQCT and analyzed the

72 characteristics of bone formation in the fracture healing process. 


\section{Materials and methods}

76

77

78

\section{Subjects}

The subjects were four patients who underwent internal fixation with a volar locking plate (VA-TCP ${ }^{\circledR}$ Two-Column Volar Distal Radius Plate 2.4, DePuy Synthes) for distal radius fractures. Their mean age was $68.8 \pm 4.9$ years, and all patients were female (Table 1). The fracture classification, based on the AO Foundation and Orthopaedic Trauma Association (AO/OTA) classification revised in 2018 [13], was 2R3A2.2 in one patient, 2R3A3.1 in two patients, and 2R3A3.2 in one patient. Splint fixation was performed until two weeks after surgery, then a wrist supporter was used until eight weeks after surgery.

The study was approved by our hospital's clinical research ethics committee (approval number: 16031418), and informed consent was obtained from the participants.

\section{HR-pQCT Scanning}

Scanning was performed within a week (baseline; 0 week), and 4, 12, and 24 weeks after fracture by using second-generation HR-pQCT (XtremeCT II, Scanco Medical, Brüttisellen, Switzerland). The scanning region was the center of the volar locking plate where there are no screws in the bone, extending $10 \mathrm{~mm}$ proximally from the most proximal point on the distal locking screw hole (Fig. 1). The scanning conditions were determined according to the manufacturer-provided standard method, with X-ray energy of $68 \mathrm{kVp}$, tube current of $1470 \mu \mathrm{A}$, integration time of $43 \mathrm{~ms}$, and number of 
97 projections of 900 , field of view of $2304 \times 2304$, and voxel size of $60.7 \mu \mathrm{m}$. The

98 scanning time was $2 \mathrm{~min}$, and the effective dose was $5 \mu \mathrm{Sv}$.

99

Fracture site identification and three-dimensional (3D) matching

101 In the baseline image, fracture lines that were included in the dorsal one-third of cortical bone were detected by two examiners and each was defined as a fracture site. In

103 this study, a total of 10 fracture sites were detected in the four patients (Fig. 2).

104 Since position and tilt change with each scan, each image needs to be matched three-

105 dimensionally for accurate comparison. The position and tilt of images 4,12 , and 24

106 weeks post-fracture were matched on a baseline image regarding cortical bone without

107 fracture or metal plate as a reference (Fig.3 A, B). 3D matching was performed using

108 bone microstructure measurement software (TRI/3D-BON: Ratoc System Engineering

109 Co., Ltd., Tokyo, Japan) so that the difference in the degree of calcification of

110 overlapping images was minimized. This method was validated in a previous study with

111 a result of acceptable precision (1.1\% of RMS\%CV) [12]. Afterward, $1 \mathrm{~mm}$ of the

112 distal and proximal ends were deleted to eliminate unmatched edge bones. Thus, 3D

113 matched images with a height of $8 \mathrm{~mm}$ at $0,4,12$, and 24 weeks post-fracture were

114 prepared (Fig.3 C).

116 Setting of regions of interest (ROI) and measurement of bone mineral density (BMD)

117 On the baseline 2D images, rectangular ROI was placed in the cortical bone defects

118 and defined as fracture site (Fx) (Fig.4 A). The same size of rectangular ROI was placed 
119 parallel on the inner and outer surface of the Fx, which was defined as inner callus (InC)

120 and external callus (ExC) (Fig.4 A). This procedure was performed from the most

121 proximal slice where fracture gap could be determined to the most distal slice

122 intermittently. By interpolating them three-dimensionally, rectangular parallelepiped

123 ROIs of Fx, InC, and ExC were made (Fig.4 B). Each ROI was used at 4, 12, and 24

124 weeks in the same coordinate systems.

125 BMD values $(\mathrm{mgHA} / \mathrm{ccm})$ were measured at each ROI (TRI/3D-BON: Ratoc System

126 Engineering Co., Ltd., Tokyo, Japan). BMD values was calculated from the X-ray

127 attenuation values by using a calibration curve prepared from hydroxyapatite phantoms.

129 Dual-energy X-ray absorptiometry (DXA)

130 The BMD values of the lumbar spine (L1-4) and femur (total hip and femoral neck)

131 were measured with DXA (Lunar Prodigy Advance, GE Lunar, Madison, WI, USA) at

13212 weeks post-fracture.

\section{Statistical analysis}

135 To assess the changes over time in BMD values and the comparison of $\mathrm{Fx}, \mathrm{InC}$, and

136 ExC, estimates, 95\% confidence intervals, and $\mathrm{p}$ values were calculated using a mixed

137 effects model. The objective variable was the log-transformed BMD value, the fixed

138 effects were taken to be site, time, baseline value, and the interaction between site and

139 time, and the random effect was taken to be the study subjects. The multiple

140 comparisons of the differences between times and between sites were done using the 
141 Tukey-Kramer HSD test. JMP statistical software (JMP ${ }^{\circledR}$ 13.0.0, SAS Institute Inc.,

142 Cary, NC, USA) was used in the statistical analysis.

143

144 


\section{Results}

146

\section{Patients' background and DXA}

148 The background and the DXA data for the patients are shown in Table 1. All four

149 patients are postmenopausal women aged 63 to 75 years old. Two out of four patients

150 had T-scores of $\leq-2.5$ (osteoporosis), and one out of four patients had T-scores of $<-$

1511.0 (osteopenia). All of the fractures occurred falling from a standing position.

\section{BMD changes between time points}

$154 \mathrm{BMD}$ values at $\mathrm{Fx}, \mathrm{InC}$, and $\mathrm{ExC}$ of $0,4,12$, and 24 weeks post-fracture and the

155 spline curves are shown in Fig.5. The BMD at InC increased in all fracture sites

156 simultaneously with the ongoing recovery at Fx. In contrast, the BMD at ExC increased

157 only in some of the fractures.

158 In Table 2, estimated BMD values and 95\% confidence intervals at $\mathrm{Fx}, \mathrm{InC}$, and $\mathrm{ExC}$

159 of $0,4,12$, and 24 weeks obtained from the mixed effects model are shown. From 0

160 week to 24 weeks, the estimated BMD values changed from 105.5 (95\% CI 98.6-113)

$161 \mathrm{mgHA} / \mathrm{ccm}$ to $428.0(95 \%$ CI $331-554) \mathrm{mgHA} / \mathrm{ccm}$ at the $\mathrm{Fx}$, from $111.0(95 \% \mathrm{CI}$

$162104-119) \mathrm{mgHA} / \mathrm{ccm}$ to $375.3(95 \%$ CI 290-486) $\mathrm{mgHA} / \mathrm{ccm}$ at the InC, and from 98.5

163 (95\% CI 91.6-106) $\mathrm{mgHA} / \mathrm{ccm}$ to 171.6 (95\% CI 132-222) $\mathrm{mgHA} / \mathrm{ccm}$ at the ExC.

164 Multiple comparisons of the estimated BMD between different time points using the

165 Tukey-Kramer HSD test (Table 2, right) showed there were significant elevations at Fx and $\mathrm{InC}$ at all times $(\mathrm{p}<0.01)$, except no significant differences were seen between 0 to 
1674 weeks. In contrast, there was no significant differences at ExC between most time

168 points, except significant differences were seen only at 4 to 24 weeks and 0 to 24 weeks $169(\mathrm{p}<0.01)$.

170

\section{$171 \quad B M D$ differences between sites}

172 From the between-site comparisons in Table 2, estimated BMD values at 0 week at

173 Fx, InC, and ExC were respectively 105.5 (95\% CI 98.6-113) mgHA/ccm, 111.0 (95\%

174 CI 104-119) mgHA/ccm, and 98.5 (95\% CI 91.6-106) mgHA/ccm, but at 24 weeks

175 they changed to 428.0 (95\% CI 331-554) mgHA/ccm, 375.3 (95\% CI 290-486)

$176 \mathrm{mgHA} / \mathrm{ccm}$, and $171.6(95 \% \mathrm{CI} 132-222) \mathrm{mgHA} / \mathrm{ccm}$.

177 On multiple comparisons using the Tukey-Kramer HSD test (Table 2, bottom), no

178 significant differences were seen between the sites at 0,4 , and 12 weeks, but at 24

179 weeks, significant differences were seen between Fx-ExC and between InC-ExC

$180(\mathrm{p}<0.01)$. This result shows that the BMD values were significantly higher at Fx and

181 InC than at ExC at 24 weeks.

182

183 Representative case

184 A representative case is shown in Fig.6. Case 2 is 63-year-old woman sustained a 185 distal radius fracture in a fall and had an AO-OTA classification of 2R3A3.2. The T-

186 scores were -0.5 for the lumbar spine (L1-L4) and 0.4 for the total hip, and she had 187 not been diagnosed with osteoporosis. On HR-pQCT images, there were large bone 
188 defects on the dorsal side at 0 week. The bone defect was gradually repaired with time 189 at 4, 12, and 24 weeks. BMD values at 0 week were Fx 95.2, InC 133.8, and ExC 66.8.

190 They changed to Fx 449.6, InC 320.5, and ExC 73.9 at 24 weeks. Whereas BMD at Fx 191 and InC increased with time, there was almost no change in BMD at ExC.

192

193 


\section{Discussion}

195 Most of distal radius fractures in women with osteoporosis is the unstable type, which

196 is commonly treated by surgical treatment with a volar locking plate in current clinical

197 practice, rather than conservative treatment with a cast. De Jong et al. and Era et al.

198 performed cadaveric studies to investigate feasibility of HR-pQCT evaluation when a

199 metal plate is placed in the distal radius $[12,14]$. In the present study, in vivo analysis

200 of the fracture healing process was performed using HR-pQCT in patients with unstable

201 distal radius fractures who underwent volar locking plate fixation.

\section{Comparison of BMD differences between sites}

BMD increased significantly with time at Fx and InC, whereas BMD increased only

slightly at ExC (Fig.5). A comparison of BMD between the sites showed BMD was significantly increased at $\mathrm{Fx}$ and $\mathrm{InC}$ compared with that at $\mathrm{ExC}$ at 24 weeks (Table 2).

In the fracture healing process, excessive interfragmentary instability will impede cartilage replacement, diminish angiogenesis and prevent bone from bridging the

209 fracture gap [15-17]. When a locking plate is used as a bridging plate that fixes only

210 main fracture segments to each other, it is said that callus formation occurs in the

211 process of bone union [2]. In the present results, although fracture is fixed with relative

212 stability using a locking plate, BMD at ExC increased only slightly. On the other hand,

213 BMD at InC increased simultaneously with BMD at Fx in all cases. There is possibility

214 that the external callus formed slightly because stability of the fracture site was mainly

215 obtained by the internal callus. Our data indicates that, in the process of bone union 
216 after osteosynthesis for distal radius fractures, the fracture site is repaired not with the

217 formation of calluses from both periosteal and endosteal sides, but usually repaired

218 gradually with the formation of callus from endosteal side alone.

219

220 Comparison of BMD changes between times

221 BMD at Fx at 24 weeks was estimated to be $428.0 \mathrm{mgHA} / \mathrm{ccm}$ (Table 2). It is

222 conjecturing from the spline curve in Fig.5, there is a possibility that this was not yet the

223 peak. In current clinical practice, bone union is often assessed with X-ray examinations.

224 Bhandari et al. stated that factors such as callus size, cortical bone continuity, and loss

225 of the fracture line detected on X-ray, pain to palpation at the fracture site, and patients'

226 weight-bearing ability were indicators for the assessment of bone union [18]. They

227 concluded that X-ray examinations are the most useful tool in assessing the fracture

228 healing process. In contrast, Watanabe et al. stated that, while X-ray examinations are

229 useful in assessing the fracture healing process, doctors make subjective assessments,

230 and there are limitations to assessing bone union with X-ray examinations [19]. In the

231 present study, fractures of all cases were judged to be bone union from X-ray

232 examinations and clinical symptoms at 24 weeks, but the BMD at the fracture site was

233 still tending to increase. The mechanical properties might not recover sufficiently, and

234 bone union might not really have obtained at 24 weeks.

235

236 Comparison with previous study 
237 With regard to the bone union process when conservative treatment is performed for

238 stable distal radius fractures, De Jong et al. reported their analysis of BMD and bone

239 microarchitecture with HR-pQCT over 2 years [11]. According to that report, cortical

240 BMD decreased until 12 weeks post-fracture and then gradually increased, while

241 trabecular BMD increased until 6 weeks post-fracture and then decreased. That study

242 measured the BMD in the entire region of distal radius in which a fracture had occurred.

243 In the present study, changes in BMD were analyzed with a focus on the fracture site

244 and surrounding areas. Different treatment and measurement region may be the reason

245 for the different results. We observed that the BMD at fracture site increased together

246 with increased BMD at endocortical site, and the increase in BMD at external site was

247 slight, suggesting the necessity of the internal callus formation in the process of bone

248 fracture repair.

$250 \quad$ Limitations

251 This study has several limitations. First, the sites that can be analyzed with this

252 method are restricted to the central part of the plate and volar side of the radius. Distal

253 sites including the main fracture line cannot be assessed due to the metal artifacts from

254 the screws. Therefore, there are limitations in judging "bone union" with HR-pQCT.

255 Also, we did not investigate unaffected side or conservatively treated patients in this

256 study.

257 Second, the number of patients was small. In fact, scans were performed in 13

258 patients, but when scanning immediately after surgery, there were motion artifacts of 
grade 3 or higher in some cases due to pain or dementia [20]. Metal artifact tends to

260 occur even with small motion. Therefore, only cases with motion artifacts of grade 1 or

2612 were used in this study. In addition, although the dorsal one-third of central plate area

262 was analyzed to avoid the effects of metal artifacts, cases in which the fracture line was

263 not included in the area were excluded. As a result, the patient number became

264 decreased to four in this study.

265 Third, the follow-up period was only 24 weeks. As shown in Fig.5, the BMD at the

266 fracture site was still increasing at 24 weeks. Therefore, we did not investigate the remodeling

267 phase of the fracture healing process in this study.

268

269 Conclusion

270 The fracture healing process of distal radius fractures treated with volar locking

271 plate were analyzed by HR-pQCT. The BMD at the inner surface of the fracture site

272 (InC) increased simultaneously with the BMD at the fracture site (Fx) in all fractures,

273 while the BMD at the outer surface of the fracture site $(\mathrm{ExC})$ increased only in some

274 fracture sites. These results indicate that bone formation on the endosteal side may be a

275 necessary condition for bone union of distal radius fractures. 


\section{Acknowledgments}

279 We would like to thank Shuntaro Sato, a specialist in biostatistics, for providing advice 280 on the statistical analyses.

281

282 Conflict of interest

283 The authors declare that they have no conflicts of interest.

284

285 


\section{References}

287 [1] Richard Marsell, Thomas A. Einhorn. THE BIOLOGY OF FRACTURE

288 HEALING. Injury. 2011 June; 42(6): 551-555. doi: 10.1016/j.injury.2011.03.031.

289 [2] Thomas P Ruedi, Richard E Buckley, Christopher G Moran. AO Principles of

290 Fracture Management Second expanded edition.

291 [3] Bonnarens F, Einhorn TA. Production of a standard closed fracture in laboratory

292 animal bone. J Orthop Res. 1984; 2(1):97-101.

293 [4] Einhorn TA. The science of fracture healing. Journal of Orthopaedic Trauma. 2005;

294 19(Suppl):S4-6.

295 [5] John A. Lynch, Mikayel Grigoryan, Anke Fierlinger, et al. Measurement of changes

296 in trabecular bone at fracture sites using X-ray CT and automated image registration and

297 processing. Journal of Orthopaedic Research 22 (2004) 362-367.

298 [6] Burghardt AJ, Link TM, Majumdar S. High-resolution computed tomography for

299 clinical imaging of bone microarchitecture. Clin Orthop Relat Res. 2011;469(8):2179-

300 2193. doi:10.1007/s11999-010-1766-x.

301 [7] Nishiyama KK, Shane E. Clinical imaging of bone microarchitecture with HR-

302 pQCT. Curr Osteoporos Rep. 2013;11(2):147-155. doi:10.1007/s11914-013-0142-7.

303 [8] Cheung AM, Adachi JD, Hanley DA, et al. High-resolution peripheral quantitative 304 computed tomography for the assessment of bone strength and structure: a review by 305 the Canadian Bone Strength Working Group. Curr Osteoporos Rep. 2013;11(2):136306 146. doi:10.1007/s11914-013-0140-9. 
307 [9] Ursina Meyer, Joost J de Jong, Sandrine GP Bours, et al. Early Changes in Bone

308 Density, Microarchitecture, Bone Resorption, and Inflammation Predict the Clinical

309 Outcome 12 Weeks After Conservatively Treated Distal Radius Fractures: An

310 Exploratory Study. Journal of Bone and Mineral Research, Vol 29, No.9, Sep 2014,

311 pp2065-2073. doi: 10.1002/jbmr.2225.

312 [10] Joost J.A. de Jong, Paul C. Willems, Jacobus J. Arts, et al. Assessment of the

313 healing process in distal radius fractures by high resolution peripheral quantitative

314 computed tomography. Bone 2014;64:65-74.

315 [11] Joost J.A. de Jong, Frans L Heyer, Jacobus JC Arts, et al. Fracture Repair in the

316 Distal Radius Postmenopausal Women: A Follow-Up 2 Years Postfracture Using

317 HRpQCT. Journal of Bone and Mineral Research, Vol 31, No.5, May 2016, pp1114-

318 1122. doi: 10.1002/jbmr.2766.

319 [12] Era M, Chiba K, Nishino Y, et al. The effects of volar locking plates for distal

320 radius fractures on the image quality of high-resolution peripheral quantitative

321 tomography. Bone 2019

322 [13] AO Foundation, ORTHOPAEDIC TRAUMA ASSOCIATION. Fracture and

323 dislocation classification compendium. Journal of Orthopaedic Trauma. Volume 32,

324 Number 1 Supplement, January 2018

325 [14] Joost J. A. de Jong, Arno Lataster, Bert van Rietbergen,et.al. Distal radius plate of

326 CFR-PEEK has minimal effect compared to titanium plates on bone parameters in high-

327 resolution peripheral quantitative computed tomography: a pilot study. BMC Medical 328 Imaging.2017 17:18. 
[15] Claes L, Eckert-Hübner K, Augat P. The effect of mechanical stability on local

330 vascularization and tissue differentiation in callus healing. J. Orthop. Res. 2002;

$33120: 1099-1105$.

332 [16] Elise F. Morgan, Kristy T. Salisbury Palomares, Ryan E. Gleason, et al.

333 Correlations between Local Strains and Tissue Phenotypes in an Experimental Model of

334 Skeletal Healing. J Biomech. 2010 August 26; 43(12): 2418-2424.

335 doi:10.1016/j.jbiomech.2010.04.019.

336 [17] Thomas A. Einhorn, Louis C. Gerstenfeld. Fracture healing: mechanism and

337 interventions. Nat Rev Rheumatol. 2015 January ;11(1): 45-54.

338 doi:10.1038/nrrheum.2014.164.

339 [18] Bhandari M, Guyatt GH, Swiontkowski MF et al. A Lack of consensus in the

340 assessment of fracture healing among orthopaedic surgeons. J Orthop Trauma 2002;

$341 \quad 16: 562-566$.

342 [19] Yoshinobu Watanabe, Yu Nishizawa, Nobuyuki Takenaka et al. Ability and

343 Limitation of Radiographic Assessment of Fracture Healing in Rats. Clin Orthop Relat

344 Res 2009 467:1981-1985 DOI 10.1007/s11999-009-0753-6.

345 [20] Pialat JB, Burghardt AJ, Sode M, Link TM, Majumdar S. Visual grading of motion

346 induced image degradation in high resolution peripheral computed tomography: impact

347 of image quality on measures of bone density and micro-architecture. Bone.

348 2012;50(1):111-118. Doi: 10. 1016/ j. bone.2011.10.003. 
$352 \quad$ Fig. 1

353 The scan region was $10 \mathrm{~mm}$ of the middle of the volar locking plate avoiding the

354 artifacts of the distal locking screws.

355

356 Fig. 2

357 Ten fracture sites were detected on the dorsal side of the cortical bone from the four

358 patients and analyzed in this study.

359

$360 \quad$ Fig. 3

$3613 \mathrm{D}$ image matching was performed at different time points $(0,4,12$, and 24 weeks $)$. A:

362 Images before 3D matching, B: Images after 3D matching with 0 week, C:

363 Superimposed images of 0 week and after 3D matching (red portions)

364

365 Fig. 4

366 Regions of interest (ROIs) of the BMD measurement are shown in 2D and 3D HR-

367 pQCT images (A and B). The fracture site (Fx) was defined as the region where cortical

368 bone continuity is lacking, the inner callus ( $\mathrm{InC})$ was defined as the inner surface of the

369 fracture site, and the external callus $(\mathrm{ExC})$ was defined as the outer surface of the

370 fracture site.

371

372 
373 Fig. 5

374 Spline curves of BMD value from 0 to 24 weeks at each site are shown. BMD at

375 Fracture site (Fx: red) increased significantly at 12 and 24 weeks, and BMD at internal

376 callus (InC: yellow) did simultaneously. On the other hand, increase of BMD at external

377 callus (ExC: blue) was smaller than the others.

378

379 Fig. 6

380 2D and 3D HR-pQCT images of a 63-year-old woman with distal radius fracture (case

381 1). There was a large bone defect on the dorsal side of distal radius. Postoperative

382 changes showed the defect was repaired over time.

383 
Table 1. Characteristics of subjects

\begin{tabular}{lccccc}
\hline Case & 1 & 2 & 3 & 4 & Mean \pm SD \\
\hline Age (years) & 63 & 65 & 72 & 75 & $68.8 \pm 4.9$ \\
Gender (male, female) & female & female & female & female & - \\
Height (m) & 1.54 & 1.47 & 1.50 & 1.52 & $1.51 \pm 0.03$ \\
Weight (kg) & 65 & 51 & 45 & 50 & $52.8 \pm 7.4$ \\
BMI (kg/m ${ }^{2}$ ) & 27.4 & 23.6 & 20.0 & 21.6 & $23.2 \pm 2.8$ \\
AO/OTA classification & $2 \mathrm{R} 3 \mathrm{~A}$ & $2 \mathrm{R} 3 \mathrm{~A}$ & $2 \mathrm{R} 3 \mathrm{~A}$ & $2 \mathrm{R} 3 \mathrm{~A}$ & \\
& 3.2 & 2.2 & 3.1 & 3.1 & - \\
& & & & & \\
Past History & none & none & none & hypertension & - \\
& & & & hypothyroidism & \\
Medication & none & none & none & Levothyroxine, & - \\
DXA T-score & & & & Alfacalcidol & \\
Lumbar Spine (L1-L4) & -0.5 & -2.3 & -2.6 & -2.4 & $-1.95 \pm 0.84$ \\
Total Hip & 0.4 & -1.4 & -1.6 & -1.6 & $-1.05 \pm 0.84$ \\
Femoral Neck & -0.3 & -1.6 & -2.4 & -2.5 & $-1.70 \pm 0.88$ \\
\hline
\end{tabular}

BMI: body mass index, DXA: dual-energy $x$-ray absorptiometry

Medication: Case 4 has taken these medicines before and after the operation. 


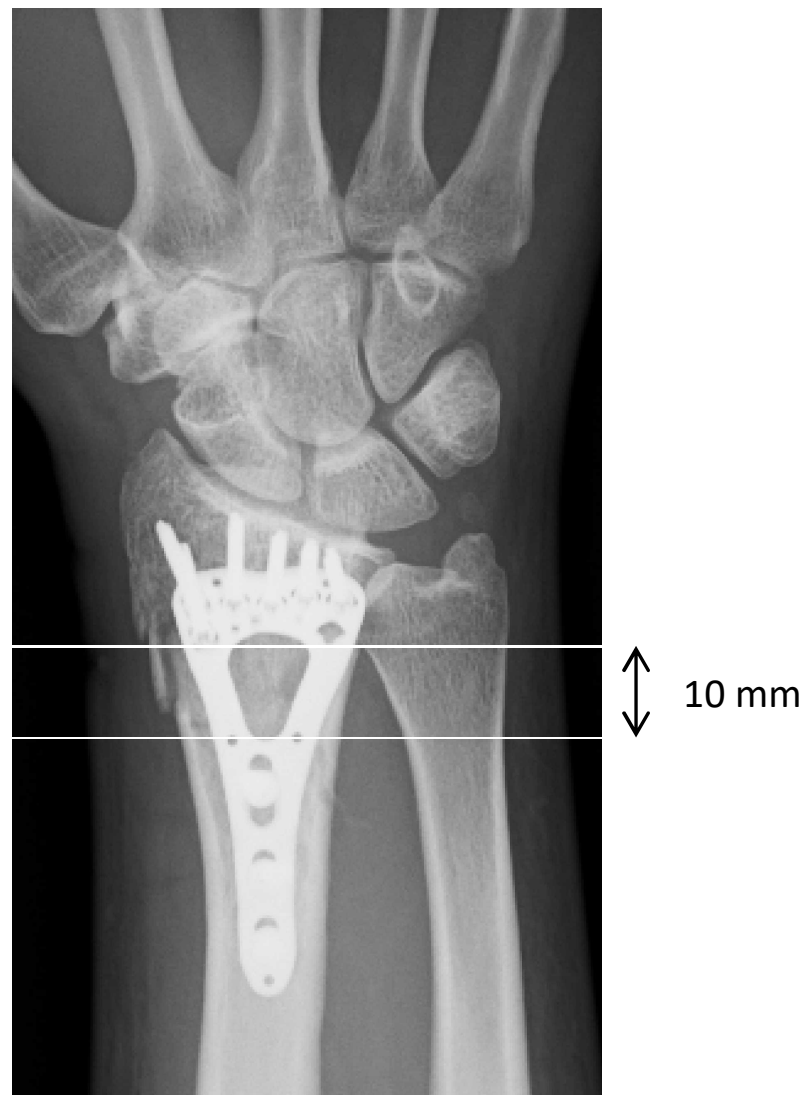

Fig. 1

The scan region was $10 \mathrm{~mm}$ of the middle of the volar locking plate avoiding the artifacts of the distal locking screws. 

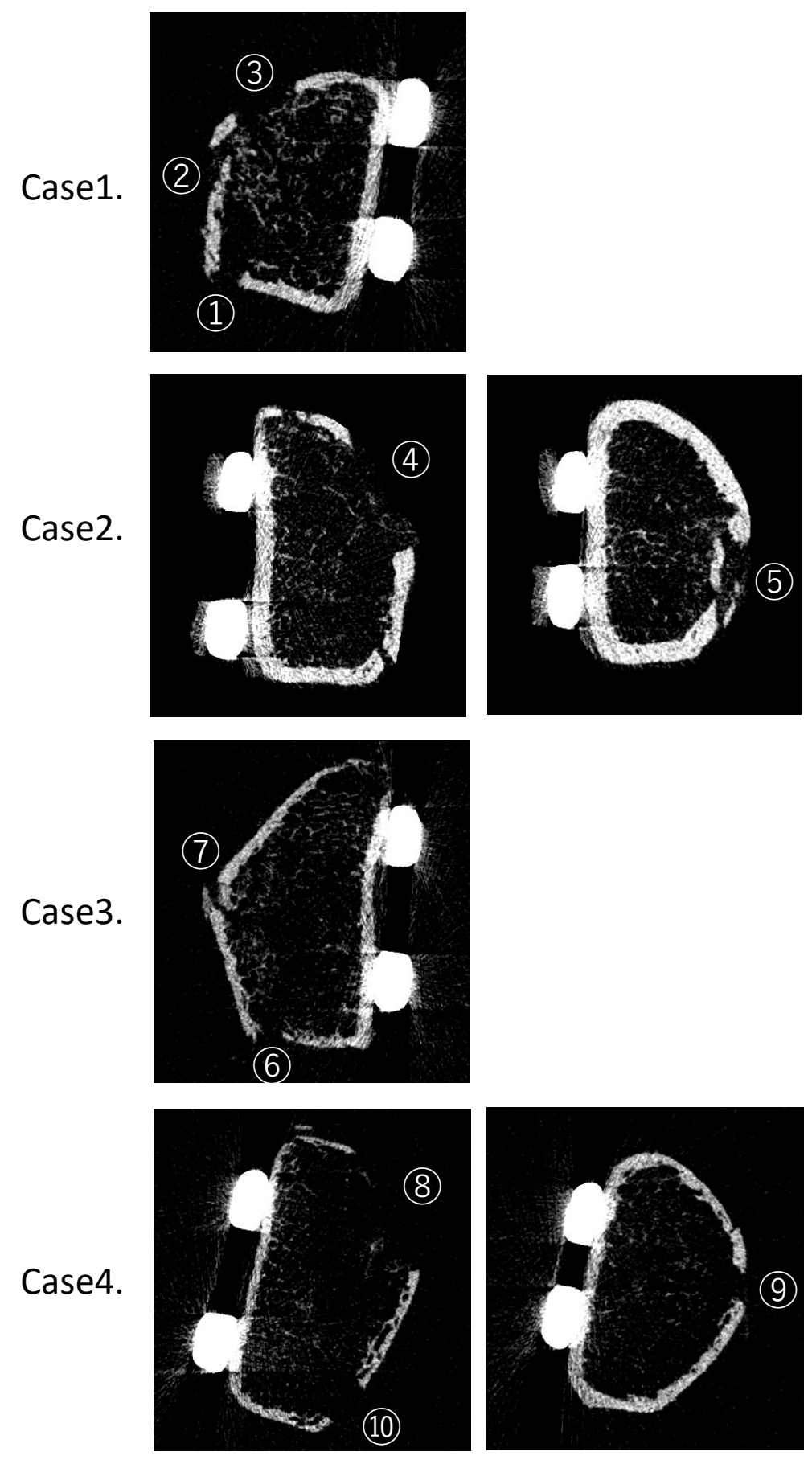

Fig. 2

Ten fracture sites were detected on the dorsal side of the cortical bone from the four patients and analyzed in this study. 


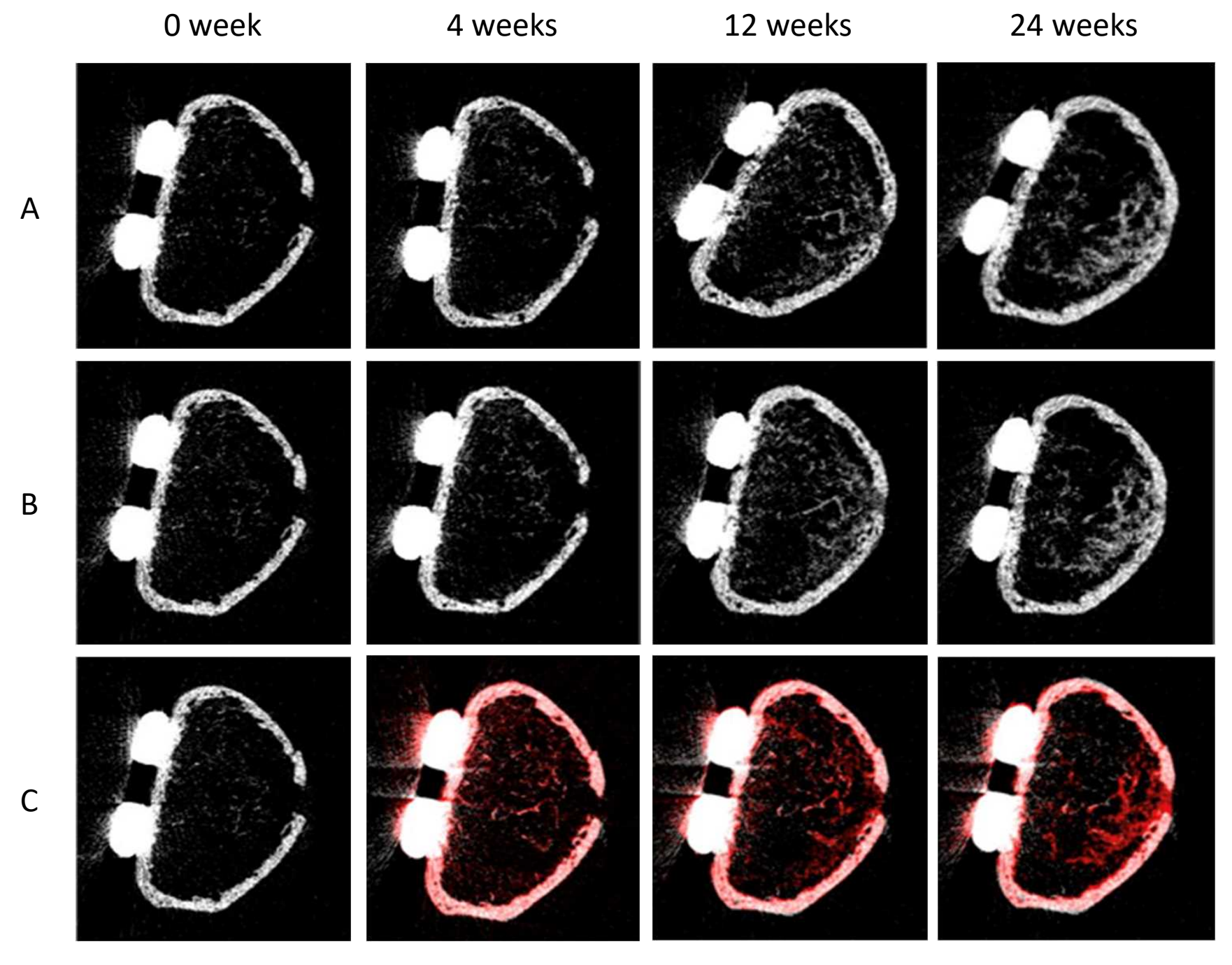

Fig. 3

3D image matching was performed at different time points $(0,4,12$, and 24 weeks).

A: Images before 3D matching,

B: Images after 3D matching with 0 week,

C: Superimposed images of 0 week and after 3D matching (red portions) 

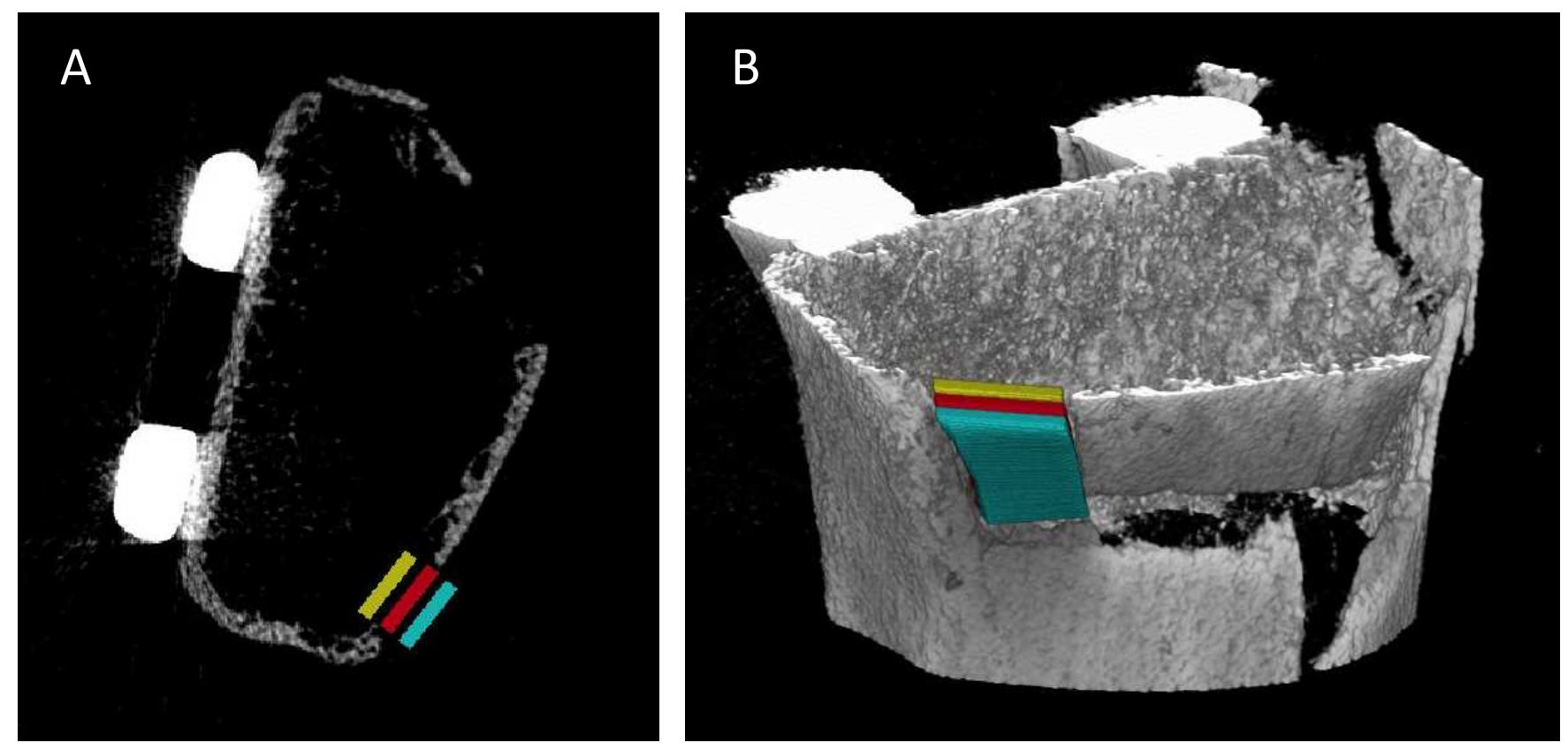

Fracture site: Fx

Inner Callus: InC

External Callus: ExC

Fig. 4

Regions of interest (ROIs) of the BMD measurement are shown in 2D and 3D HR-pQCT images $(A$ and $B)$. The fracture site $(F x)$ was defined as the region where cortical bone continuity is lacking, the inner callus $(\operatorname{InC})$ was defined as the inner surface of the fracture site, and the external callus (ExC) was defined as the outer surface of the fracture site. 


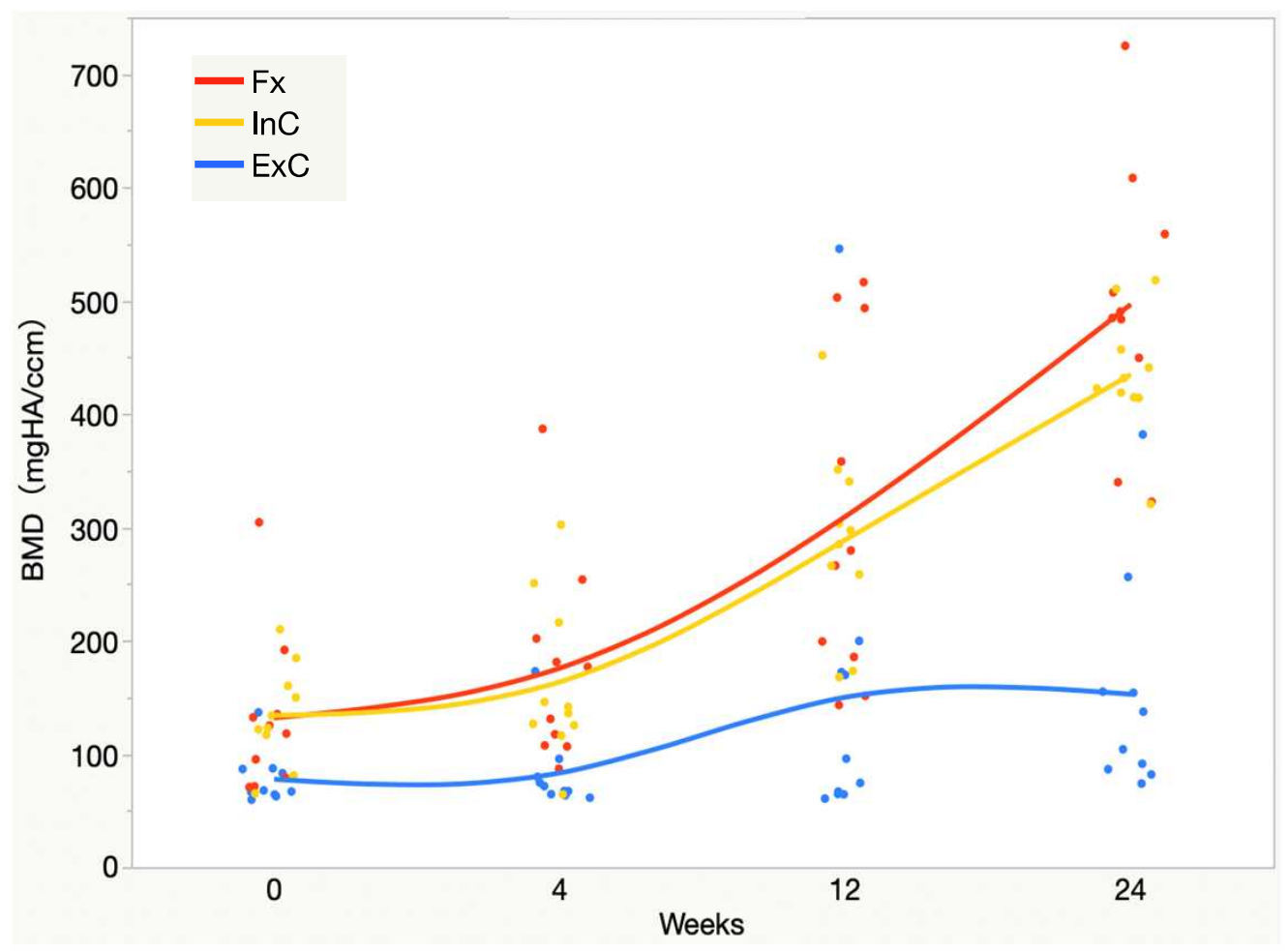

\section{Fig. 5}

Spline curves of BMD value from 0 to 24 weeks at each site are shown. BMD at Fracture site (Fx: red) increased significantly at 12 and 24 weeks, and BMD at internal callus (InC: yellow) did simultaneously. On the other hand, increase of BMD at external callus (ExC: blue) was smaller than the others. 
Table 2 BMD values at fracture site ( $F x)$, inner callus ( $I n C)$, and external callus (ExC) from 0 to 24 weeks

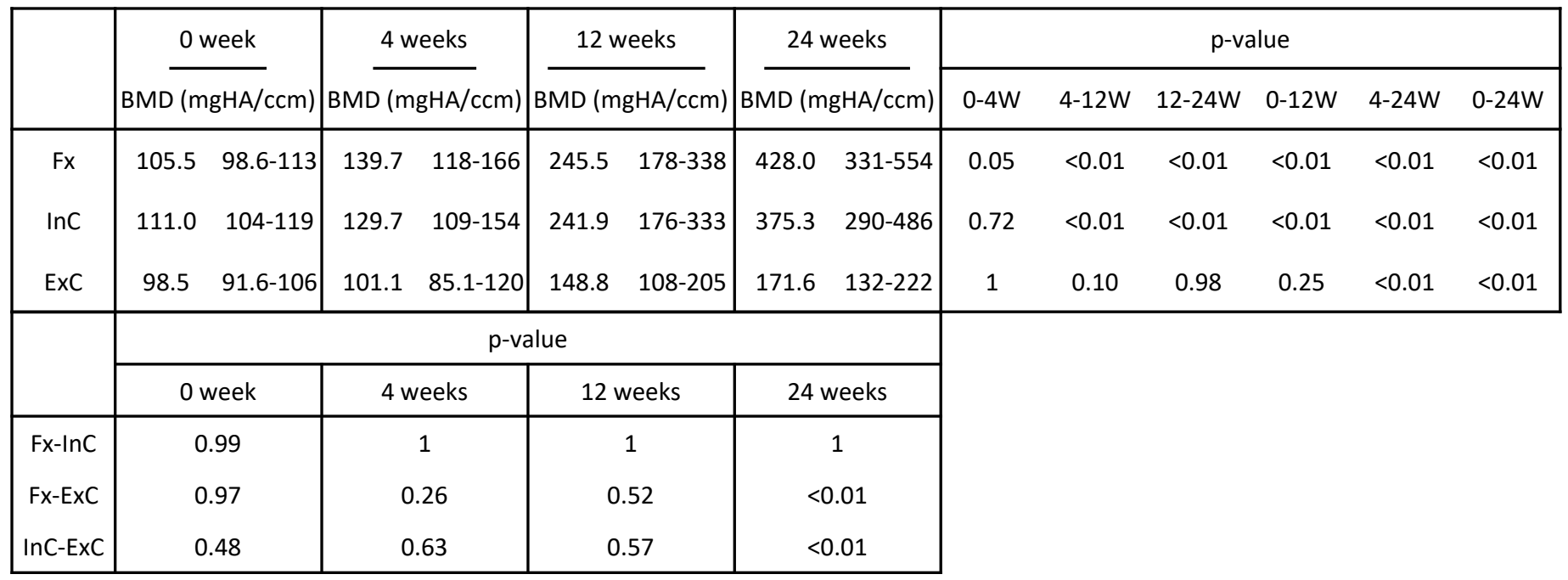

Estimated values and $95 \%$ confidence intervals are shown. $p$ values were calculated from the mixed effects model. Multiple comparisons of differences between times and between groups were done using the Tukey-Kramer HSD test. 
0 week
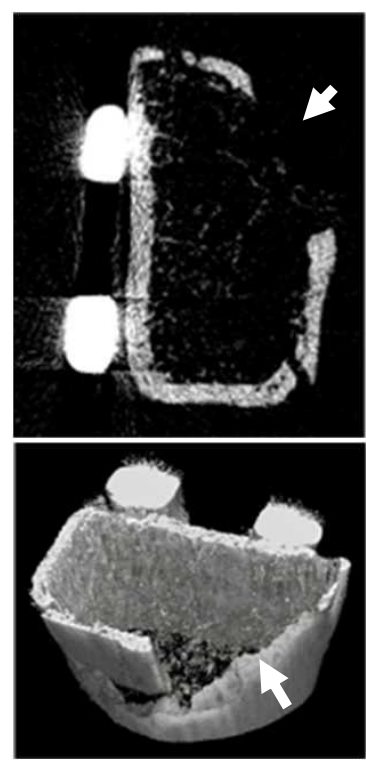

4 weeks
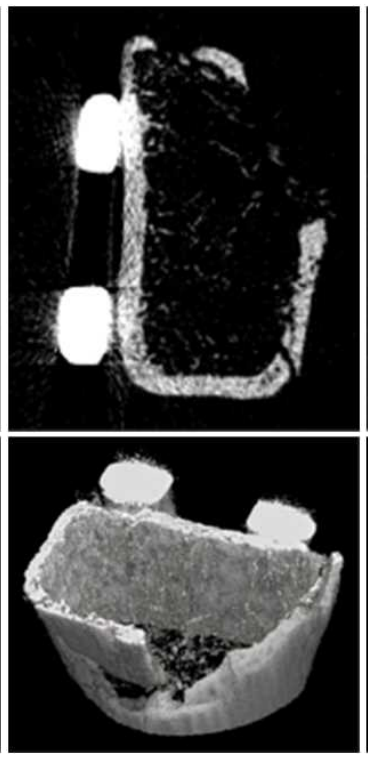

12 weeks
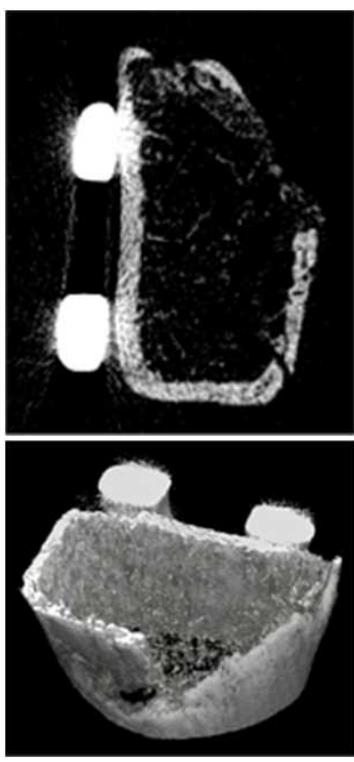

24 weeks
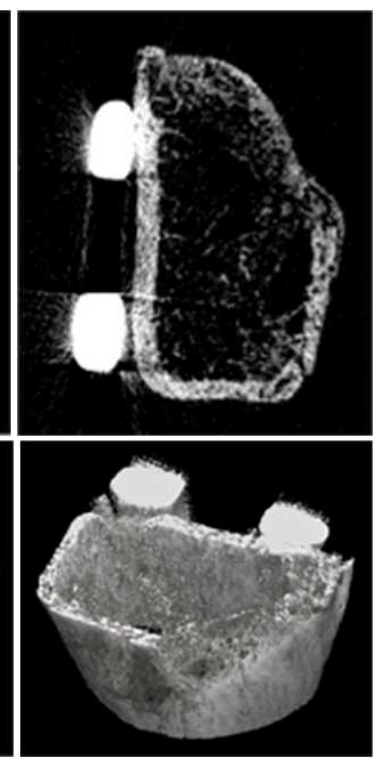

Fig. 6

2D and 3D HR-pQCT images of a 63-year-old woman with distal radius fracture (case 1). There was a large bone defect on the dorsal side of distal radius. Postoperative changes showed the defect was repaired over time. 